\title{
Capacidad de absorción del conocimiento, aprendizaje y tecnologías de la información en las organizaciones: estado del arte y evolución de la investigación
}

\section{Knowledge absorption capacity, learning and information technologies in organizations: state of the art and evolution of research}

\author{
Yasser Abarca Sánchez, ${ }^{\text {la }}$ y Urbi Barreto Rivera ${ }^{2}$ \\ Universidad Nacional San Antonio Abad del Cusco, Perú ${ }^{12}$ \\ D Orcid ID: https://orcid.org/0000-0001-7941-9346 ${ }^{1}$ \\ (iD) Orcid ID: https://orcid.org/0000-0002-2205-6799²
}

Recibido: 20 de abril de 2020

Aceptado: 28 de octubre de 2020

\begin{abstract}
Resumen
Esta investigación tiene por objetivo realizar el estado del arte de la capacidad de absorción del conocimiento, el aprendizaje y las tecnologías de la información (TI) en las organizaciones; las referencias estudiadas formulan relaciones entre las variables antes mencionadas de manera incipiente, por lo que el presente artículo sistematiza la información y propone una representación gráfica de complementariedad entre estas. La metodología empleada se basa en una revisión literaria, tipo sistémica, aplicando una búsqueda bibliométrica de las variables utilizando las bases de datos Scopus y Science Direct por medio de un análisis documentario, con un proceso analíticosintético basado en la categorización de artículos acerca de la capacidad de absorción del conocimiento, capacidad de absorción del conocimiento y aprendizaje y finalmente capacidad de absorción del conocimiento y tecnologías de la información, utilizando para la selección final de artículos, criterios basados en población, unidad de análisis y año de publicación. Se tiene como resultado la elaboración de representaciones gráficas que esquematizan las variables de forma independiente, así como la relación entre ellas.
\end{abstract}

Palabras clave: Capacidad de absorción del conocimiento, adquisición del conocimiento, aprendizaje, TI, gestión del conocimiento.

\footnotetext{
Abstract

${ }^{a}$ Correspondencia al autor

E-mail: yasser.abarca@unsaac.edu.pe
} 
This research aims to carry out the state of the art of the absorption capacity of knowledge, learning and information technologies (IT) in organizations; The references studied formulate relationships between the aforementioned variables in an incipient manner, so this article systematizes the information and proposes a graphical representation of complementarity between them. The methodology used is based on a systemic literary review, applying a bibliometric search of the variables using the Scopus and Science Direct databases by means of a documentary analysis, with an analytical-synthetic process based on the categorization of the capacity of absorption of knowledge, capacity for absorption of knowledge and learning and finally capacity for absorption of knowledge and information technologies, using criteria based on population, unit of analysis and year of publication for the final selection of articles. The result is the elaboration of graphical representations that outline the variables independently, as well as the relationship between them.

Keywords: Knowledge absorption capacity, knowledge acquisition, learning, IT, knowledge management

\section{Introducción}

La capacidad de las organizaciones para absorber nuevo conocimiento del exterior es crucial para la innovación y desarrollo, se constituye como un sustento de ventajas competitivas dentro de un contexto donde la globalización de los mercados, los rápidos cambios tecnológicos, la reducción del ciclo de vida tecnológico y la creciente agresividad de los competidores han cambiado las reglas del juego en la gestión de organizaciones (Camisón \& Fores, 2014). De acuerdo a Roberts, Galluch, Dinger, \& Grover (2012) una organización absorbe el conocimiento del exterior solamente si los repositorios de conocimiento y los cerebros de los miembros individuales son capaces de buscar y ser receptivos a ese conocimiento en base a los conocimientos previos. Posteriormente, el conocimiento fluye a través de la organización, este flujo de conocimiento puede ser facilitado por estructuras y procesos apropiados (Roberts et al., 2012) que pueden crear mecanismos eficientes para aplicar el conocimiento a propósitos útiles.

En ese entender, todas las organizaciones aprenden, ya sea que elijan conscientemente o no, es un requisito fundamental para su existencia sostenida. Algunas empresas promueven deliberadamente el aprendizaje organizacional, desarrollando capacidades que son consistentes con sus objetivos; otros no hacen ningún esfuerzo enfocado y, por lo tanto, adquieren hábitos que son contraproducentes (Lerch, Seitz, \& Wagner, 2012).

Es así que, la evolución de la tecnología de información (TI), últimamente ha producido diversos impactos que han sido de gran importancia para el hombre, cuyo avance ha alcanzado a 
todos, hoy es una realidad que la última generación ha desarrollado más formas de capacidad de transferir el conocimiento (Van der Heiden, Pohl, Mansor, \& van Genderen, 2016). Es claro el rol que juega las nuevas tecnologías de comunicación e información en el mundo y más que nada, en el desarrollo de los países, por tanto, es una herramienta fundamental que favorece a un cambio positivo en las organizaciones siempre y cuando sean utilizadas de forma ética y creativa (Sánchez Duarte, 2008).

El valor de esta investigación radica en sintetizar la aplicación de la capacidad de absorción del conocimiento, el aprendizaje y las TI para demostrar su poder como una capacidad organizativa clave a través de una búsqueda bibliométrica. El artículo está dividido en dos secciones principales. La primera parte describe los orígenes y evolución en la literatura organizacional de la capacidad de absorción del conocimiento, el aprendizaje y las TI. En la segunda parte, se revisa cómo se relacionan las variables antes mencionadas.

\section{Metodología}

Para el cumplimiento del objetivo planteado, se realizó una revisión literaria, de forma sistémica, basada en un resumen de evidencias realizada por un experto o un grupo de expertos para un determinado tema (Whittemore, Chao, Jang, Minges, \& Park, 2014), utilizando la base de datos Scopus y Science Direct con las palabras claves: capacidad de absorción, aprendizaje y tecnologías de información, donde se identificó, evaluó y sintetizó los estudios para desarrollar el estudio del arte y así obtener conclusiones de los datos recopilados (Moja et al., 2005). Para ello se utilizó la técnica del análisis documental, con un procesamiento analítico - sintético donde se describe y representa los documentos incluyendo la clasificación, extracción y traducción de los mismos (Dulzaides Iglesias \& Molina Gómez, 2004), de esa forma se hicieron representaciones gráficas referentes a nuestro estudio, todo esto a través de una búsqueda bibliométrica el cual se desarrolla en el siguiente punto.

\section{Enfoque preliminar y búsqueda bibliométrica}

La técnica empleada fue la búsqueda bibliométrica en las bases de datos científicas Scopus y Science Direct que tuvo una estructura de búsqueda utilizando los siguientes códigos de campo para la variable capacidad de absorción y luego su relación con tecnologías de información y aprendizaje siendo el siguiente: 
TITLE-ABS-KEY ( absorptive AND capacity ), TITLE-ABS-KEY ( absorptive AND capacity AND information AND technology ), TITLE-ABS-KEY ( absorptive AND capacity AND learning )

Luego se procedió a realizar el filtro correspondiente con los siguientes códigos de campo:

(LIMIT-TO ( EXACTSRCTITLE , “Journal Of Business Research”)

OR LIMIT-TO ( EXACTSRCTITLE, "International Journal Of Innovation Management")

OR LIMIT-TO ( EXACTSRCTITLE, "Journal Of Knowledge Management")

OR LIMIT-TO (EXACTSRCTITLE, "Technological Forecasting And Social Change")

OR LIMIT-TO ( EXACTSRCTITLE, “Strategic Management Journal”)

AND ( LIMIT TO ( DOCTYPE , "ar") )

AND ( LIMITTO ( SUBJAREA , "BUSI") )

AND ( LIMIT-TO ( EXACTKEYWORD , "Absorptive Capacity") )

AND ( LIMIT-TO ( SRCTYPE, “j”) ).

El método de búsqueda se basó en encontrar artículos científicos relacionados con las palabras clave: capacidad de absorción del conocimiento, aprendizaje y TI. El área temática elegida para esta búsqueda se enmarcó en negocios y gestión, el criterio de elección responde a la cantidad de citas y el factor de impacto de las revistas durante el período comprendido entre 1958 y 2019.

En base a los criterios antes mencionados, se encontraron 3192 artículos que se observan en la tabla 1. Para la selección inicial de los documentos se tuvo en cuenta las palabras clave y el tipo de documento (artículos científicos) en el área de estudio de negocios y gestión siendo un total de 977 estudios. Luego se crearon estándares para juzgar la relevancia de los artículos por tema: (1) estrechamente relacionado, (2) moderadamente relacionado y (3) ligeramente relacionado; los artículos que cayeron en la categoría (3) fueron excluidos, obteniendo una cantidad de 296 estudios. Finalmente se evaluó la importancia de la contribución en 3 niveles: (1) muy importante, (2) moderadamente importante, y (3) poco importante; en este caso, los documentos que caen en la clase (3) también fueron excluidos, obteniendo una cantidad de 209 estudios. El criterio utilizado para seleccionar los artículos según la escala planteada se basó en la, cantidad de población, unidad de análisis y año de publicación. 


\section{Tabla 1}

Etapas para la selección de trabajos

Escenario

Búsqueda bibliométrica

Primera selección: tipo de documento y tema.

Segunda selección: nivel de relevancia

Tercera selección: nivel de importancia

Total, artículos analizados

\section{Número de artículos}

3.192

977

296

209

209

\section{Fuente: Elaboración propia}

Para clasificar adecuadamente estos 209 documentos, fue necesario establecer una categorización de artículos de las siguientes variables, de forma individual y relacionada,: capacidad de absorción del conocimiento, capacidad de absorción del conocimiento y aprendizaje y finalmente capacidad de absorción del conocimiento y tecnologías de la información y comunicaciones como se observa en la tabla 2. La fase de extracción de datos incluyó el análisis cuantitativo de los 209 artículos e incluyó información sobre: Tendencias de la investigación, autores con mayor número de publicaciones, revistas con el mayor número de publicaciones y países de origen de la investigación. Aunque la búsqueda a través de las diferentes bases de datos abarcó desde 1958 hasta el 2019, el primer resultado se publicó en 1986; no fue hasta el año 2014 cuando comenzó a publicarse un número significativo de artículos que trataban esta relación de variables. La producción a partir de entonces aumentó con ligeras variaciones. Entre 2013 y 2019, más del 80\% del trabajo fue publicado, siendo 2016 el año con el mayor número de contribuciones. El interés en esta área de estudio comenzó con la capacidad de absorción en el año 1986. A partir de 2003, comenzaron a aparecer sobre aspectos generales de la relación entre capacidad de absorción y las otras variables. 
Tabla 2

Tendencias de investigación por categoría

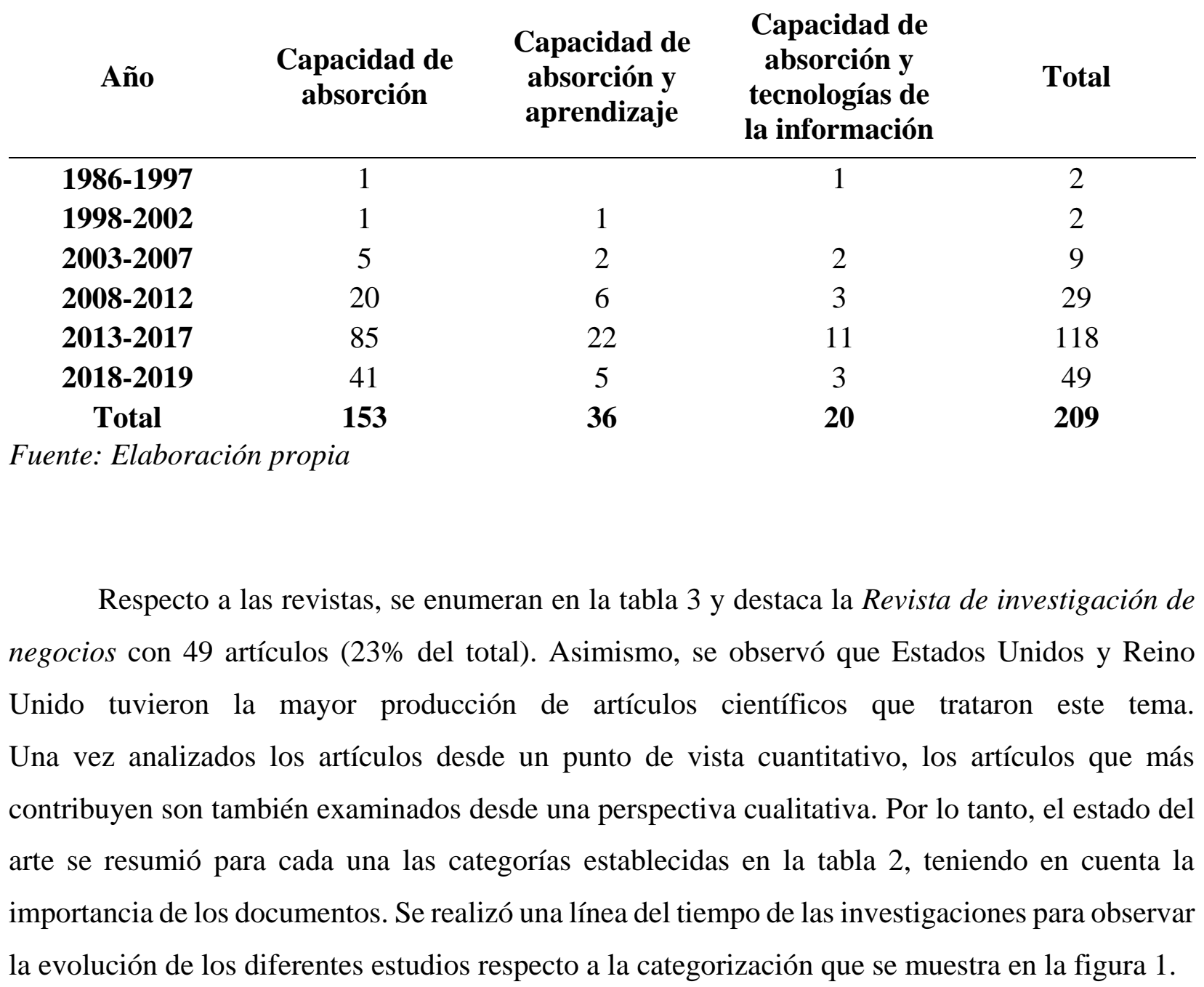


Tabla 3

Tendencias de investigación por categoría

\begin{tabular}{lcc}
\multicolumn{1}{c}{ Revistas } & Numero de artículos & Porcentaje \\
\hline Journal of Business Research & 49 & $23 \%$ \\
Journal of Knowledge Management & 44 & $21 \%$ \\
Technological Forecasting and Social Change. & 43 & $21 \%$ \\
International Journal of Innovation & 41 & $20 \%$ \\
Management & & \\
Strategic Management Journal & 32 & $15 \%$ \\
Fuente: Elaboración propia & &
\end{tabular}

Figura 1

Evolución de investigaciones

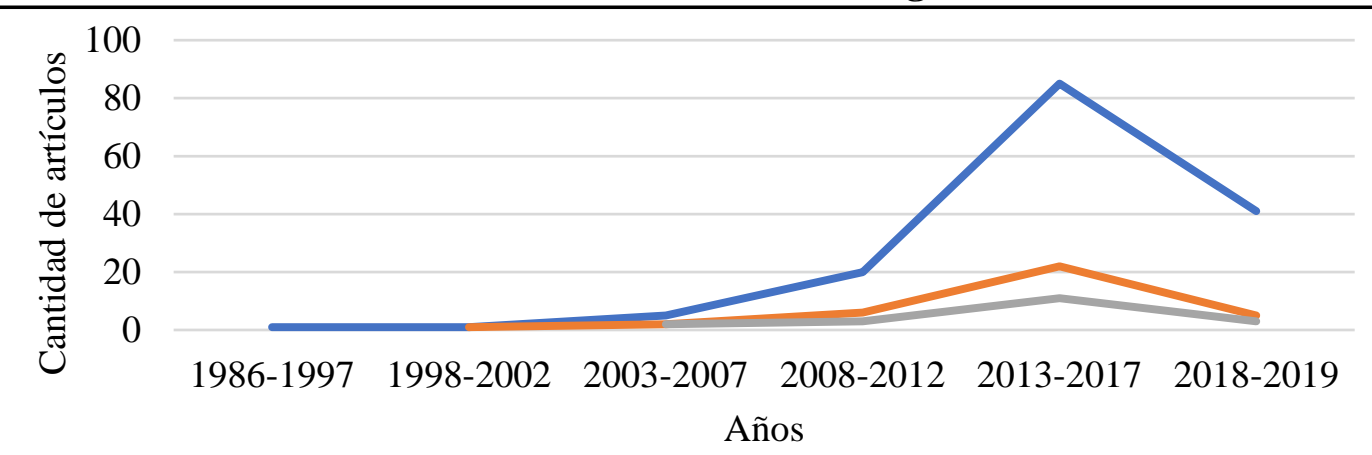

Capacidad de absorción del conocimiento

Capacidad de absorción del concimiento y aprendizaje

Capacidad de absorción del conocimiento y tecnologías de la información

Fuente: Elaboración propia basado en base de datos Scopus y Science Direct. 


\section{Estado del arte}

El concepto de capacidad de absorción se introdujo en las investigaciones de gestión en medio de un creciente interés en el mundo científico y empresarial acerca de aprendizaje organizacional, alianzas estratégicas y la visión de la empresa basada en los recursos (Aribi \& Dupouët, 2016; García Montijo \& Inés León Balderrama, 2015; Lane, Koka, \& Pathak, 2006). De acuerdo a Denford \& Ferris (2018) se distinguen tres tipos de capacidades en la gestión del conocimiento: capacidad de absorción, capacidad combinativa y capacidad de deserción, demostrando que dichas capacidades ayudan a igualar las brechas de conocimiento y las existencias de conocimiento tácito y explícito dentro de las empresas, entre empresas y socios.

La capacidad de absorción de por si es difícil de definir debido a que sus conceptos varían considerablemente, evolucionando y cambiando significativamente a lo largo del tiempo, por tanto, el poder y los límites son características centrales de una visión de proceso de la capacidad de absorción (Easterby-Smith, Graça, Antonacopoulou, \& Ferdinand, 2008), asimismo, estudios previos sobre el desarrollo de la memoria sugieren que los conocimientos previos acumulados aumentan tanto la capacidad de poner nuevos conocimientos en la memoria, llamado adquisición de conocimientos como la capacidad de recordar y usar dichos conocimientos (Cohen \& Levinthal, 1990; Stock, Greis, \& Fischer, 2001); quiere decir que, la capacidad de absorción no es solo una cuestión de ósmosis: depende de la voluntad deliberada y la agencia individual (García Montijo \& Inés León Balderrama, 2015); sin embargo, si se busca dar un significado, esta puede entenderse como los conocimientos previos que la organización necesita para asimilar y descubrir nuevos conocimientos (Lau \& Lo, 2015; Wang \& Han, 2011).

Las dimensiones de la capacidad de absorción vienen a ser la adquisición, la asimilación, la transformación y la explotación tal como se muestra en la figura 2 los cuales conforman procesos de aprendizaje distintos en una organización (Zahra \& George, 2002), también facilitan el uso de instrucciones claras y estrictas para la absorción de conocimiento optimizando el proceso de desglosar las capacidades de socialización existentes (Frans A. J., Henk W., \& Michiel de, 2015). Otros investigadores de diversas disciplinas han podido reconocer el poder de la capacidad de absorción y se espera que el uso futuro de este concepto muestre un mayor reconocimiento de sus múltiples dimensiones y sus enlaces para crear y sostener una ventaja competitiva (Zahra \& George, 2002). 


\section{Figura 2}

\section{Dimensiones de la capacidad de absorción}

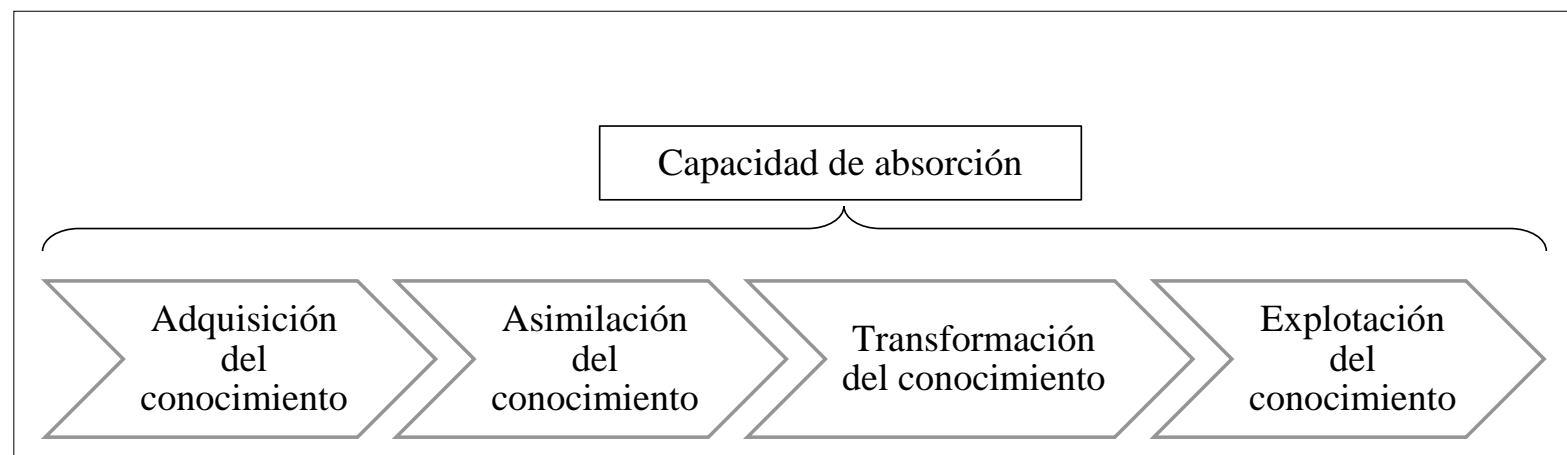

Fuente: Elaboración propia basado en Zahra \& George (2002).

\section{La capacidad de absorción y el entorno}

El entorno de una organización está relacionado con la diversificación en los mercados, para ello se requiere la adquisición de mayores conocimientos, un factor crítico que facilita la absorción de conocimiento es la presencia de conocimiento previo dentro de la organización (Cohen \& Levinthal, 1990), lo que a su vez puede reducir los costos de la diversificación; dicho de otro modo, a medida que disminuye la relación con los mercados, no solo es probable que aumente la necesidad de adquisición de conocimiento, sino que también disminuya el nivel de conocimiento anterior, lo que aumenta los costos de la diversificación (Kumar \& Seth, 2001). Por tanto, significa que la capacidad de valorar y asimilar nuevos conocimientos externos, se relacionará con la capacidad de aplicarlos comercialmente para lograr los objetivos de la organización (Lane \& Lubatkin, 2017).

Entendiendo el efecto de la diversificación de mercados se observa que, el conocimiento interno de un nuevo mercado puede requerir una capacidad de absorción de conocimiento adicional, ya que la organización puede necesitar reconocer y asimilar continuamente la información necesaria relacionada con el nuevo mercado (Cohen \& Levinthal, 1990); sin embargo, mientras que la diversificación requiere capacidad de absorción para que la organización pueda desarrollar y combinar el conocimiento internamente, es probable que las empresas conjuntas requieran un nivel de capacidad de absorción relativamente menor (Kumar \& Seth, 2001). 
Es así que, por la complejidad que presenta la diversificación de mercados Lewin, Massini, \& Peeters (2011) mencionan las rutinas en una organización y la evolución que tienen en la misma definidas como meta-rutinas, estas últimas regulan cambios en rutinas de nivel inferior como la tensión adaptativa, tasas de innovación y cambio, y la interacción con el entorno sociopolítico y económico externo. (Nelson \& Winter, 1982). Del mismo modo se establecen mecanismos organizativos asociados con las capacidades de coordinación para aumentar principalmente la capacidad de absorción potencial, mientras que los mecanismos organizacionales asociados con las capacidades de socialización fortalecen principalmente los aspectos reales de capacidad de absorción realizada (Zahra \& George, 2002)

Sin embargo, estos mecanismos organizacionales tienen efecto en algunas dimensiones de la capacidad de absorción del conocimiento como en el incremento de la transformación de nuevos conocimientos externos los cuales; sin embargo, no están relacionados con la explotación del mismo. Esto sugiere que la explotación requiere estructuras de conocimiento más estables y densamente conectadas que la transformación (Jansen, Van den Bosch, \& Volberda, 2005). Cuando la competencia se centra más en el conocimiento, una organización debe desarrollar una comprensión profunda de su propio conocimiento, los procesos que utiliza para convertir el conocimiento en capacidades y la capacidad de esas capacidades para satisfacer al entorno (Lane \& Lubatkin, 2017).

\section{Adquisición del conocimiento}

La adquisición de conocimiento externo refleja la función de identificación, que representa el generador de inteligencia para la organización. Las señales ambientales externas se identifican y la información sobre esas señales se recopila y transmite a través de los límites de la organización. Cuanto más conocimiento se pueda recopilar durante un período determinado, mejor funcionará la capacidad de adquisición. Cuanta más información recopile la organización a través del proceso de búsqueda, más opciones habrá para identificar cambios en el entorno y, por lo tanto, mejor será el rendimiento de la empresa. (Liao, Welsch, \& Stoica, 2003)

\section{Asimilación del conocimiento}

La asimilación del conocimiento se refiere a las actividades y procesos de la organización los cuales permiten analizar, procesar, interpretar y comprender la información obtenida de fuentes externas. Aquellas ideas y descubrimientos que se encuentran fuera de la zona de búsqueda de una 
organización no se toman en cuenta porque la organización no tiene una comprensión sólida. (Flor Peris, Oltra Mestre, \& García Palao, 2011).

\section{Transformación del conocimiento}

Consiste en la comunicación del conocimiento generado a todos los departamentos e individuos relevantes en una organización, por ello una diseminación exitosa requerirá flujos de conocimiento significativos e intercambio para asegurar que el conocimiento llegue a las personas relevantes. La organización debe estar bien estructurada para que las redes tanto formales como informales se maximicen para transferir el conocimiento dentro de la organización y entre los diferentes departamentos funcionales (Liao et al., 2003).

\section{Explotación del conocimiento}

La explotación del conocimiento se centra en aquellas actividades que permiten a las organizaciones perfeccionar, extender y aprovechar las competencias existentes y además establecer nuevas competencias a través del conocimiento adquirido, asimilado y transformado en sus operaciones, es decir, el enfoque de la explotación está en la conversión del conocimiento en nuevos productos (Zahra \& George, 2002).

\section{Tecnologías de la información}

Las organizaciones están bajo una presión constante para crear sinergias en los recursos bajo su control. Las TI y la gestión del conocimiento son dos áreas que a menudo son fructíferas para proporcionar un mayor valor cuando se administran con cuidado, es por eso que la tecnología a menudo juega un papel de apoyo en el proceso de aprendizaje (Mulamula \& Amadi-Echendu, 2016; Sánchez Duarte, 2008).

Se entenderá las TI como una oportunidad tecnológica, es decir, a las posibilidades de progreso tecnológico en diferentes industrias; es decir, cuán fácil, en términos de tiempo y costos, es lograr innovaciones en un campo de conocimiento determinado (Nieto \& Quevedo, 2005); sin embargo, esa oportunidad puede estar entendida en la facilidad de uso percibida y la utilidad percibida, entendiendo de esta forma la capacidad de detectar oportunidades de innovación y aprovechar esas oportunidades competitivas del mercado al reunir los activos, el conocimiento y las relaciones necesarios con rapidez y sorpresa (Sambamurthy, Bharadwaj and, \& Grover, 2013), 
así como lo que las personas realmente hacen con el artefacto tecnológico en sus prácticas recurrentes y situadas (Orlikowski, 2000).

Entonces las TI ejercen una influencia crucial sobre el tipo y la variedad de los resultados tecnológicos alcanzados por las organizaciones, esto es especialmente cierto con respecto al nivel de gasto en investigación y desarrollo teniendo en cuenta que las personas forman juicios de utilidad percibida en parte comparando cognitivamente lo que un sistema es capaz de hacer con lo que necesitan hacer en su trabajo (García-Morales, Ruiz-Moreno, \& Llorens-Montes, 2007; Venkatesh, Davis, \& Venkatesh, 2000), es así que el diseño de la ciencia participa en la creación de artefactos tecnológicos que impactan a personas y organizaciones, al mismo tiempo tiene una visión simplista de los mismos ya que solo se centra en la resolución de problemas (Hevner, March, Park, \& Ram, 2013). Siguiendo a Wade \& Hulland (2004), se conceptualiza tres tipos de capacidades de TI, de afuera hacia adentro, de adentro hacia afuera y de expansión, las que son:

a) Las capacidades de TI de afuera hacia adentro son exteriores y facilitan la capacidad de identificación de conocimiento de una empresa.

b) Las capacidades de TI de adentro hacia afuera están enfocadas hacia el interior y aumentan la capacidad de aplicación de conocimiento de una empresa.

c) Las capacidades de TI de expansión o abarcadoras integran las capacidades de entrada y salida de una empresa

\section{Aprendizaje}

La definición de aprender se concibe como la adquisición de conocimientos, teniendo como soporte la memorización más repetitiva que comprensiva, que se aplica a diversas situaciones; esta definición permite entender que el aprendizaje organizacional a medida que crece la organización empieza a diferenciarse del aprendizaje individual, como también la forma o evolución del modo de capturar el aprendizaje de sus miembros de forma individual (Kim, 1998). De igual modo el aprendizaje se refiere al proceso de las actividades de gestión, intercambio, adquisición y exploración de conocimientos para mejorar el desempeño de las tareas (Chou, 2020), dando paso al enfoque sociocognitivo del aprendizaje que se centra en un aprendizaje individual y colectivo donde la organización influye en la forma de como el individuo percibe un problema, diseña la solución y produce la acción para solucionar (Wiewiora, Chang, \& Smidt, 2020). 
Las organizaciones están compuestas por individuos, pueden aprender independientemente de cualquier individuo específico, pero no independientemente de todos los individuos (Kim, 1998), aún se sabe relativamente poco sobre la mente humana y el proceso de aprendizaje, parece que cuanto más conocimiento se tiene, el individuo más se da cuenta de lo poco que sabe. Las organizaciones aprenden a través de sus miembros y el aprendizaje individual es un factor indispensable para cumplir con este hecho; sin embargo, esto no es suficiente para que exista una retención colectiva de ese conocimiento, por ello se debe entender de cómo el conocimiento individual es transferido a la organización (Serrat, 2017).

Kim (1998), distingue entre lo que se aprende y la comprensión, así como, el uso que se hace de este conocimiento. Estos dos niveles quedan definidos en los términos: aprendizaje operacional y conceptual. Siendo el primer nivel aquel que se adquiere a nivel de proceso, a través del cual el individuo aprende los pasos requeridos para llevar a cabo ciertas tareas (Pérez González \& Portuondo Padrón, 1997), es decir un reconocimiento preconsciente del patrón y / o posibilidades inherentes a un flujo personal de experiencia, explicando a través de palabras y / o acciones, de una idea o idea para uno mismo y para los demás (Crossan, Lane, \& White, 1999), asimismo Zollo \& Winter (2013) observan que este proceso resulta en ajustes adaptativos a los conjuntos de rutinas existentes o en mejoras de la necesidad de un cambio más fundamental. El segundo nivel incluye el pensamiento sobre las causas subyacentes de las acciones requeridas en la organización cuyas condiciones, procedimientos y conceptos son debatidos y se crean nuevos marcos de referencias (Kim, 1998), debido a que fuerzas de socialización, educativa y organizacional configuran el desarrollo de un estilo de aprendizaje particular y especializado (Kolb \& Kolb, 2014), centrándose en la atención y las competencias limitadas.

Es así que los dos niveles mencionados anteriormente se relacionan de forma análoga hoy en día con el aprendizaje reflexivo (Feindt \& Weiland, 2018) donde los niveles que se identifican son conceptualizados como aprendizaje de primer orden y de segundo orden (Koole, 2020), siendo el primero un aprendizaje que ocurre constantemente mientras nos encontramos en un sistema social complejo y el segundo un aprendizaje que se refiere a la resolución de problemas a través de la racionalidad instrumental la cual generó nuevos problemas. De este modo Grin (2016) define el aprendizaje reflexivo como un aprendizaje de posibles cambios en las prácticas, así como en las estructuras cambiantes. Por ello es que el aprendizaje ha ganado la atención de los académicos 
debido a sus transiciones de sostenibilidad, ya que este tiene como elemento a la confianza que se relaciona con el aprendizaje reflexivo (Goyal \& Howlett, 2020).

Finalmente se puede mencionar dentro de todo este proceso a la adaptabilidad del aprendizaje que se refiere a como el individuo se adecua dentro de una organización para incrementar su aprendizaje. Se enfoca principalmente en el desempeño adaptativo, la flexibilidad de roles y la capacidad de autogestión en el aprendizaje, por tanto, se infiere que la adaptabilidad del aprendizaje es un factor importante de las capacidades individuales para la absorción del conocimiento (Gebauer, Worch, \& Truffer, 2012; Newman \& Chaharbaghi, 2000), esto se observa en la figura 3.

Figura 3

\section{La adaptabilidad del aprendizaje como factor de la capacidad de absorción}

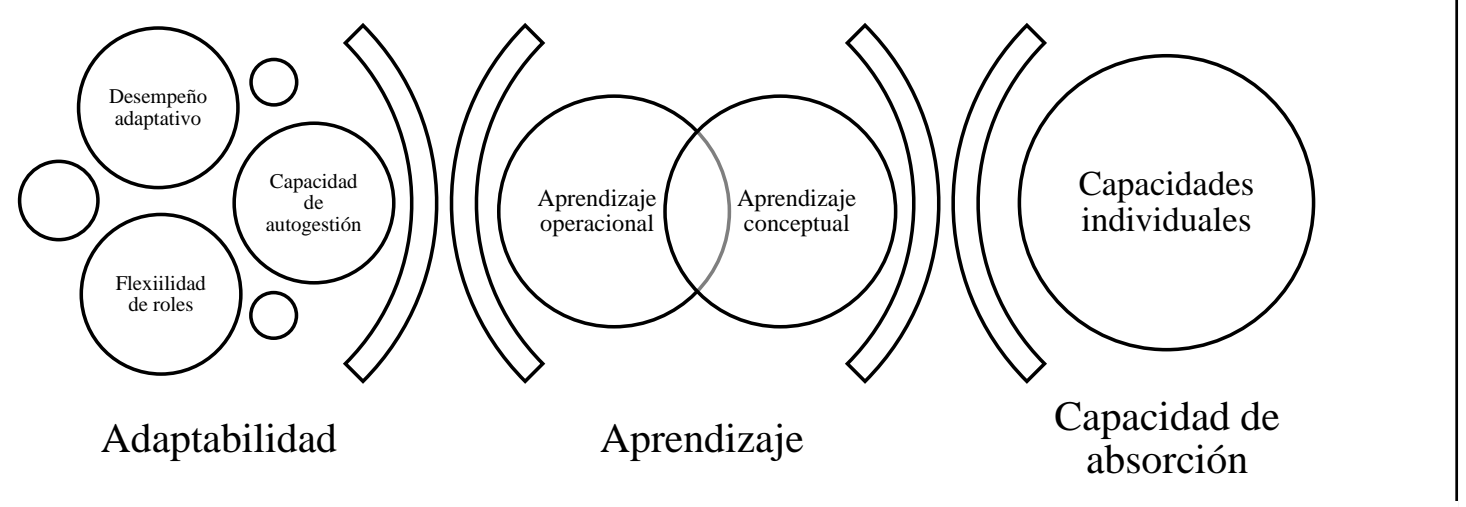

Fuente: Elaboración propia basada en Kim (1998), y Newman \& Chaharbaghi (2000).

\section{Capacidad de absorción y aprendizaje}

La capacidad de absorción de una organización se basará en la inversión previa del desarrollo de sus componentes al igual que en las capacidades de absorción de los individuos es así que, la capacidad de absorción organizativa tenderá a desarrollarse acumulativamente (Cohen \& Levinthal, 1990); sin embargo, el nivel de comunicación y el intercambio entre las áreas funcionales en una organización son altos, lo que conduce a un nivel relativamente alto de 
capacidad de absorción. (Gebauer et al., 2012; Li, Huang, \& Lin, 2014). La capacidad de absorción es importante desde una perspectiva empresarial y organizativa más amplia, debido a su enfoque en la adquisición y uso de información externa, el papel de la capacidad de absorción en las alianzas entre empresas es un área natural de investigación (Stock et al., 2001), por tanto, la flexibilidad organizacional juega un papel muy importante debido a que promueve el flujo de intercambio de información y conocimiento entre los trabajadores de una empresa mejorando la capacidad de absorción (Martins, 2014).

Bradshaw, Langley y Simon (1983) mencionan que la capacidad de aprendizaje implica el desarrollo de la capacidad de asimilar el conocimiento existente, es por ello que el entorno ofrecerá una base más sólida para el aprendizaje debido a que aumentará la posibilidad de que la información entrante, que es información potencialmente útil que se genera por la incertidumbre en los dominios del conocimiento (Cohen \& Levinthal, 1990; Thorne, 2003), se relacione con lo que ya se conoce.

De igual forma Cohen y Levinthal, (1990) mencionan que la capacidad de absorción de una organización no depende simplemente de la interfaz directa de la organización con el entorno externo sino también depende de las transferencias de conocimiento a través y dentro de las subunidades que pueden estar bastante alejadas del punto de entrada original, por tanto, la forma en que complementan los procesos de aprendizaje exploratorio, transformador y explotador tienen un efecto directo para crear y mantener una ventaja competitiva a través de desarrollo de la capacidad de absorción (Lichtenthaler, 2009).

De acuerdo a las investigaciones de Cohen y Levinthal (1990), se adopta la conceptualización acerca de la capacidad de absorción potencial, este tipo de capacidad de absorción está formado por los procesos de adquisición del conocimiento externo y de asimilación del conocimiento, este último se refiere a las rutinas y procesos de la empresa que le permiten analizar, procesar, interpretar y comprender la información obtenida de fuentes externas; entonces la capacidad de absorción potencial hace a la empresa receptiva para adquirir y asimilar conocimiento externo, pero no garantiza la explotación de este conocimiento, ya que para ello se requiere la capacidad de absorción realizada (Peris et al., 2011).

Nieto y Quevedo, (2005) hace referencia a la capacidad de absorción variable, que representa el vínculo entre el know-how generado fuera de la empresa y el conocimiento obtenido 
internamente, este punto significa que un análisis de las relaciones entre las variables estructurales y el comportamiento innovador de las empresas podría enriquecerse con la inclusión de alguna variable interna que incorpore la capacidad de aprendizaje, con lo que las empresas enfrentan las oportunidades que brinda el entorno cercano; sin embargo Lichtenthaler (2009), menciona que puede existir rendimientos marginales bajos en el aprendizaje por lo cual es necesario crear un equilibrio entre la capacidad de absorción y las capacidades de innovación interna (figura 4).

Figura 4.

Relación entre la capacidad de absorción y el aprendizaje

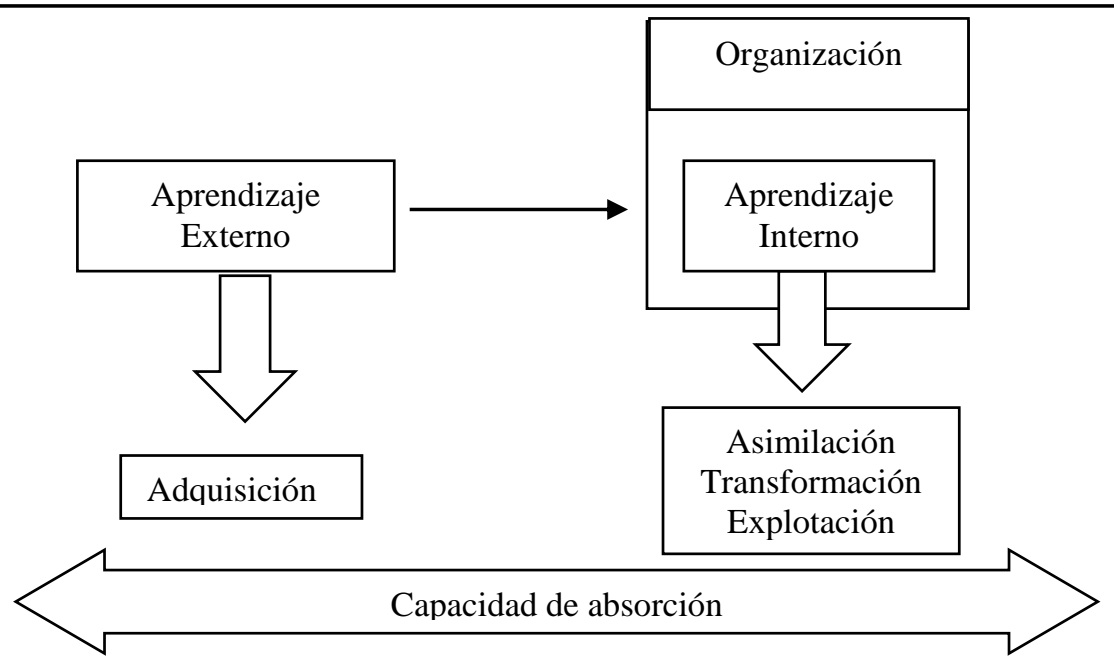

Fuente: Elaboración propia basada en Flor Peris et al. (2011), y Nieto \& Quevedo (2005)

\section{Capacidad de absorción y tecnologías de la información}

El conocimiento organizacional depende de procesos más tácitos, como la difusión, para ser transferidos a la tecnología, las estructuras y las rutinas que retienen el conocimiento para el uso organizativo futuro, es así que Jones (2017) menciona tres aspectos que son importantes para el uso de intercambio de conocimiento para una innovación tecnológica: la confianza, comunicación y capacitación en tecnología, siendo esta última la responsable de enseñar a recopilar conocimientos y aprender sobre las innovaciones tecnológicas, sin embargo, no solo debe enfocarse en la innovación (Cuen-Michel \& Ramíres-Romero, 2013), sino también en los socios de investigación que participan en la transferencia tecnológica de colaboración (Lerch et al., 2012; Nooteboom \& Gilsing, 2006). 
Por ello, aunque la tecnología funciona como estrategia de la innovación, la adquisición de tecnología debe utilizarse con algunas condiciones, considerando la capacidad de absorción requerida, ya que puede generar un impacto negativo sin la presencia de dichas capacidades (Huang \& Rice, 2009; Lin, Chang, \& Chang, 2004). Asimismo, Sánchez Duarte (2008) sugiere una variedad de acciones organizativas que pueden mitigar las limitaciones de capacidad de absorción en equipos virtuales los cuales son, oportunidades de creación de experiencia con miembros del equipo, tecnología y tareas, acceso a herramientas que apoyan el trabajo altamente interdependiente, desarrollo de estrategias y tecnologías que soporten memoria transactiva, desarrollo de estrategias y tecnologías que apoyen la transferencia de conocimiento tácito, entre otros. Asimismo Newman \& Chaharbaghi, (2000) mencionan el impacto que tienen estos factores para el desarrollo de la capacidad de absorción debido a que en la organización no solo se debe considerar las tecnologías para la base de conocimiento sino también deben considerar a los manejadores de tecnología creando un equilibrio entre ambos.

De acuerdo a Roberts, Galluch, Dinger, \& Grover (2012), la capacidad de absorción desempeña un papel en varias corrientes importantes de la investigación de sistemas de información; como el conocimiento de TI de negocios, transferencia de conocimiento, asimilación de TI y valor de negocio de TI, sin embargo, la capacidad de absorción se ha conceptualizado y medido de diversas maneras, dejando su rol más amplio ambiguo e infrautilizado en la red nomológica de un sistema de información, a esta referencia se suma lo mencionado por Nooteboom \& Gilsing (2006) acerca de la distancia tecnológica, que indica el nivel de conocimiento y la actualización constante que se tiene acerca de las nuevas tendencias en TI, donde la capacidad de absorción disminuye con la distancia tecnológica, y el valor de la novedad aumenta con ella como se puede observar en la figura 5. 
Figura 5.

\section{Relación entre la capacidad de absorción y tecnologías de información}

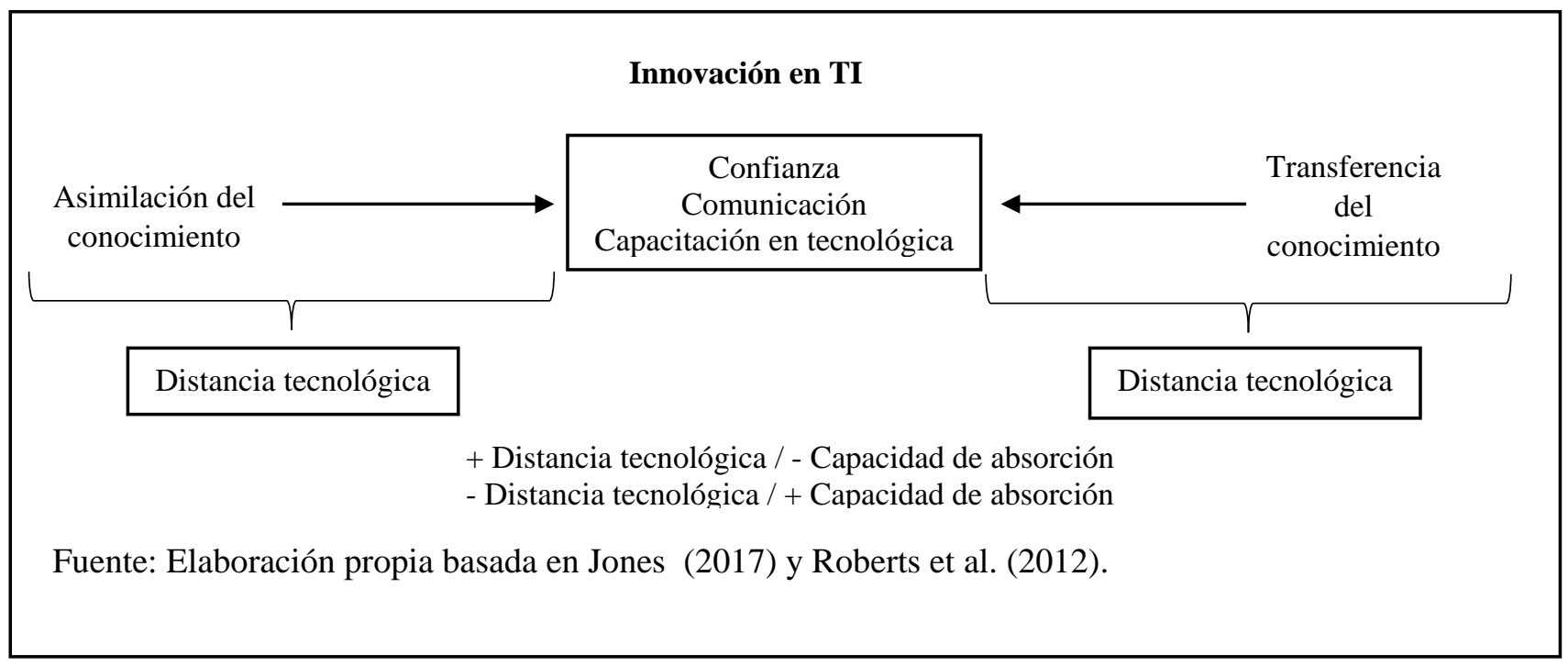

\section{Resultados y discusión}

La capacidad de absorción del conocimiento se logra a través de conocimientos previos, los cuales servirán para poder asimilar y descubrir nuevos conocimientos del entorno, logrando el incremento del mismo (Cohen \& Levinthal, 1990; Lau \& Lo, 2015; Stock et al., 2001; Wang \& Han, 2011). Las dimensiones que la conforman son: la adquisición del conocimiento que se enfoca en la recopilación de información externa (Liao et al., 2003), la asimilación del conocimiento que permite analizar, procesar, interpretar y comprender la información externa obtenida (Flor Peris et al., 2011), la transformación del conocimiento que se encarga de la comunicación del nuevo conocimiento generado a toda la organización (Liao et al., 2003) y finalmente la explotación del conocimiento que consiste en plasmar el nuevo conocimiento en productos o procesos tangibles (Zahra \& George, 2002).

Por otro lado, las tecnologías de la información son vitales en el proceso de innovación respecto a costo y tiempo para poder gestionar el conocimiento (Mulamula \& Amadi-Echendu, 2016; Nieto \& Quevedo, 2005), tres aspectos que participan en este proceso son: la confianza del conocimiento que tiene la organización, es decir, que tan confiable es la fuente de origen de la información así como el proceso de obtención de la misma, la forma en que se realiza la 
comunicación a través de toda la organización y finalmente la capacitación tecnológica que enseña a utilizar las diversas TI que servirán para la recopilación de información (Cuen Michel \& Ramíres Romero, 2013; Jones, 2017); sin embargo, la distancia tecnológica que menciona Nooteboom \& Gilsing (2006), que es el nivel de conocimiento acerca de la evolución de las TI, es un factor que determinará si el proceso de innovación tecnológica se realizará eficientemente.

Asimismo, el aprendizaje se divide en dos en relación a una organización: la capacidad de aprendizaje externa, que se basa en la adquisición de conocimiento que ofrece el entorno, y el aprendizaje interno que se enfoca en la asimilación, transformación y explotación del conocimiento que ya se tiene (Cohen \& Levinthal, 1990), ambos tipos de aprendizaje se desarrollan en base a la capacidad de absorción del conocimiento para lograr una ventaja competitiva. De la misma manera, se tiene niveles de aprendizaje: el aprendizaje operacional que se consigue a través de un patrón de secuencias establecidas para un proceso determinado o rutinas existentes en una organización (Kim, 1998; Pérez González \& Portuondo Padrón, 1997), y el aprendizaje conceptual, el cual se obtiene a través de debates en relación a condiciones, procedimientos y conceptos dentro de una organización y así crear una base para el aprendizaje particular y especializado (Kim, 1998; Kolb \& Kolb, 2014).

Conforme a lo expuesto anteriormente, la capacidad de absorción del conocimiento que tiene por objetivo incrementar el mismo es gestionada por TI para ejecutar de forma eficiente todas sus dimensiones las cuales son: adquisición, asimilación, transformación y explotación del conocimiento (Cohen \& Levinthal, 1990; Lau \& Lo, 2015; Stock et al., 2001; Wang \& Han, 2011; Zahra \& George, 2002). La información obtenida a través del uso de las TI establecidas en el proceso de absorción del conocimiento que sirve para un aprendizaje externo o interno, será de valor para la organización si se toma en cuenta tres factores para una correcta innovación en las TI que son: confianza, comunicación y capacitación tecnológica; todo este proceso será más eficiente siempre y cuando la distancia tecnológica sea menor, incrementando el aprendizaje en sus dos niveles operacional y conceptual (Kim, 1998; Kolb \& Kolb, 2014; Mulamula \& Amadi-Echendu, 2016; Nieto \& Quevedo, 2005; Nooteboom \& Gilsing, 2006), todo este proceso se muestra en la figura 6. 
Figura 6.

Relación entre la capacidad de absorción y aprendizaje a través de las tecnologías de la información
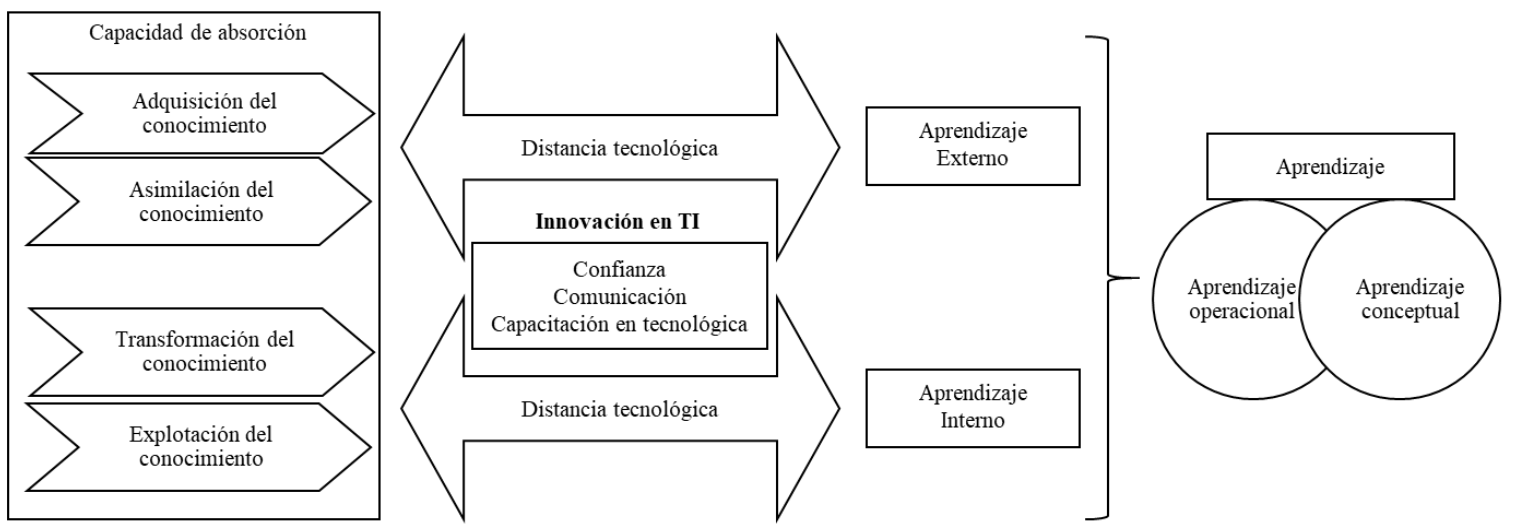

Fuente: Elaboración propia basada en Zahra \& George (2002), Kim (1998), Newman \& Chaharbaghi (2000), Flor Peris et al. (2011), Nieto \& Quevedo (2005), Jones (2017) y Roberts et al. (2012).

\section{Conclusiones}

Se realizó una revisión bibliográfica de las variables donde se encontró una variedad de artículos que mencionaban y describían la relación entre ellas, tomando como base de datos científicas a Scopus y Science Direct, realizando filtros y seleccionando los artículos con mayor relevancia para obtener literatura especializada de las variables de investigación.

Se observa como estas variables han ido evolucionando con el tiempo, el primer artículo que menciona a la capacidad de absorción del conocimiento fue publicado en el año 1986, a partir del año 2003 se ve una tendencia de estudiar esta variable conjuntamente con otras como el aprendizaje y las tecnologías de la información, siendo esta última la que tiene menor cantidad de estudios en el área de gestión y negocios, asimismo se observa que la bibliografía relacionada con las tres variables conjuntas es escasa.

La capacidad de absorción es una capacidad dinámica que se encuentra dentro de los procesos de una organización para adquirir, asimilar, transformar y explotar el conocimiento, teniendo en cuenta el conocimiento previo que se posee. Se define este concepto en base a los 
autores Zahra y George (2002), Cohen y Levinthal (1990), Lane \&yLubatkin (2017), Frans A. J. et al. (2015) y P. J. Lane et al. (2006).

El aprendizaje se entiende como la combinación del aprendizaje organizacional y el aprendizaje individual, de este modo se puede concebir como un medio principal para lograr la renovación estratégica de una empresa adquiriendo el conocimiento a través de la experiencia. Se define este concepto en base a los autores H. W. Lane y White (1999), Zollo y Winter (2013) y Kim (1998).

Las TI son definidas por la actitud hacia el uso que se le quiere dar, siendo los efectos la facilidad de uso que se percibe respecto a la TI y la utilidad que pueda tener en la organización, dando lugar a una oportunidad tecnológica que ejerza un impacto sobre el tipo y la variedad de los resultados tecnológicos obtenidos por las organizaciones. Se define este concepto en base a los autores Orlikowski (2000), Sambamurthy et al. (2013) y Venkatesh et al. (2000).

Se observa como la adquisición, asimilación, transformación y explotación del conocimiento logran un impacto en el nivel de aprendizaje operacional y conceptual a través de la distancia tecnológica la cual será mayor o menor dependiendo de la innovación tecnológica que tenga la organización para un ambiente externo e interno. Teniendo en cuenta la revisión de la literatura anterior y las representaciones gráficas realizadas, se propone un diseño de la relación que tiene la capacidad de absorción, el aprendizaje y las tecnologías de información.

Es así que el aprendizaje no solo se logra por el uso particular de TI o simplemente por aplicar el proceso de adquisición de conocimiento, ya que esto sería un proceso incompleto, la relación correcta entre las dimensiones de la capacidad de absorción de conocimiento, y las TI dan como resultado el aprendizaje deseado en todos sus niveles.

\section{Referencias}

Aribi, A., \& Dupouët, O. (2016). Absorptive capacity: A non-linear process. Knowledge Management Research and Practice, 14(1), 15-26. https://doi.org/10.1057/kmrp.2014.17

Bradshaw, G. F., Langley, P. W., \& Simon, H. A. (1983). Studying scientific discovery by computer simulation. Science, 222(4627), 971-975. https://doi.org/10.1126/science.222.4627.971 
Camisón, C., \& Fores, B. (2014). Capacidad de Absorción: Antecedentes y resultados. Strategic Management, 391, 13-22. Retrieved from http://repositori.uji.es/xmlui/bitstream/handle/10234/125745/65177 PDF.pdf?sequence $=3 \&$ is Allowed $=\mathrm{y}$

Chou, S. W. (2020). Understanding relational virtual community members' satisfaction from a social learning perspective. Journal of Knowledge Management, 24(6), 1425-1443. https://doi.org/10.1108/JKM-12-2019-0683

Cohen, W. M., \& Levinthal, D. A. (1990). Absorptive Capacity: A New Perspective on Learning and Innovation. Administrative Science Quarterly, 35(1), 128. https://doi.org/10.2307/2393553

Crossan, M. M., Lane, H. W., \& White, R. E. (1999). An organizational learning framework : From intuition to institution. Academy of Management, 24(3). https://doi.org/10.5465/amr.1999.2202135

Cuen Michel, C., \& Ramíres Romero, J. L. (2013). Usos, funciones y efectos de las TIC en el aprendizaje de una licenciatura en Ciencias de la Comunicación. Edutec. Retrieved from https://www.uned.ac.cr/academica/edutec/memoria/ponencias/cuen_ramirez_133.pdf

Denford, J. S., \& Ferriss, A. (2018). Absorption, combination and desorption: knowledgeoriented boundary spanning capacities. Journal of Knowledge Management, 22(7), 14251441. https://doi.org/10.1108/JKM-08-2017-0325

Dulzaides Iglesias, M. E., \& Molina Gómez, A. M. (2004). Análisis documental y de información: Dos componentes de un mismo proceso. Acimed, 12(2).

Easterby-Smith, M., Graça, M., Antonacopoulou, E., \& Ferdinand, J. (2008). Absorptive capacity: A process perspective. Management Learning, 39(5), 483-501. https://doi.org/10.1177/1350507608096037

Feindt, P. H., \& Weiland, S. (2018). Reflexive governance: exploring the concept and assessing its critical potential for sustainable development. Introduction to the special issue. Taylor \& Francis.

Flor Peris, M. L., Oltra Mestre, M. J., \& García Palao, C. (2011). La relación entre la capacidad 
de absorción del conocimiento externo y la estrategia empresarial: un análisis exploratorio. Revista Europea de Direccion y Economía de La Empresa, 20(1), 69-88. Retrieved from https://core.ac.uk/download/pdf/61401361.pdf

Frans A. J., V. den B., Henk W., V., \& Michiel de, B. (2015). Co-evolution of Firm Absorptive Capacity and Knowledge Environment: Organizational Forms and Combinative Capabilities. Organization Science Publication, 10(5), 551-568. https://doi.org/10.1287/orsc.10.5.551

García-Morales, V. J., Ruiz-Moreno, A., \& Llorens-Montes, F. J. (2007). Effects of technology absorptive capacity and technology proactivity on organizational learning, innovation and performance: An empirical examination. Technology Analysis and Strategic Management. https://doi.org/10.1080/09537320701403540

García Montijo, L., \& Inés León Balderrama, J. (2015). La capacidad de absorción del conocimiento y sus dimensiones en Pymes Acuícolas: el caso Sonora, México. Revista AquaTIC, 43, 14-22. Retrieved from http://www.revistaaquatic.com

Gebauer, H., Worch, H., \& Truffer, B. (2012). Absorptive capacity, learning processes and combinative capabilities as determinants of strategic innovation. European Management Journal, 30(1), 57-73. https://doi.org/10.1016/j.emj.2011.10.004

Goyal, N., \& Howlett, M. (2020). Who learns what in sustainability transitions? Environmental Innovation and Societal Transitions, 34, 311-321.

Grin, J. (2016). Transition Studies: Basic Ideas and Analytical Approaches. In Handbook on Sustainability Transition and Sustainable Peace (pp. 105-121). Springer.

Hevner, A. R., March, S. T., Park, J., \& Ram, S. (2013). Design Science in Information Systems Research. MIS Quarterly, 28(1), 75-105. https://doi.org/10.2307 / 25148625

Huang, F., \& Rice, J. (2009). The role of absorptive capacity in facilitating "open innovation" outcomes: A study of Australian SMEs in the manufacturing sector, 13(2), 201-220. https://doi.org/10.1142/S1363919609002261

Jansen, J. J. P., Van den Bosch, F. A. J., \& Volberda, H. W. (2005). Managing Potential and realized absorptive capacity: How do organizational antecedents matter. Transplantation 
Proceedings, 48(6), 999-1015. https://doi.org/10.1016/j.transproceed.2011.12.061

Jones, G. E. (2017). Knowledge sharing and technological innovation : The effectiveness of trust , training , and good communication. Cogent Business \& Management, 11(1). https://doi.org/10.1080/23311975.2017.1387958

Kim, D. H. (1998). The Link between Individual and Organizational Learning. The Strategic Management of Intellectual Capital, 41-62. https://doi.org/10.1016/B978-0-7506-98504.50006-3

Kolb, A. Y., \& Kolb, D. A. (2014). Learning Styles and Learning Spaces : Enhancing Experiential Learning in Higher Education. Source: Academy of Management Learning \& Education, 4(2), 193-212. https://doi.org/10.5465/amle.2005.17268566

Koole, B. (2020). Trusting to learn and learning to trust. A framework for analyzing the interactions of trust and learning in arrangements dedicated to instigating social change. Technological Forecasting and Social Change, 161(August 2019), 120260. https://doi.org/10.1016/j.techfore.2020.120260

Kumar, S., \& Seth, A. (2001). Knowledge, absorptive capacity, and the theory of the diversified firm. Academy of Management Proceedings, (1959), 1-7. https://doi.org/10.5465/APBPP.2001.6123191

Lane, P. J., Koka, B. R., \& Pathak, S. (2006). The reification of absorptive capacity: A critical review and rejuvenation of the construct. Academy of Management Review, 31(4), 833-863. https://doi.org/10.5465/AMR.2006.22527456

Lane, P. J., \& Lubatkin, M. H. (2017). Relative absorptive capacity and interorganizational learning. Strategic Management, 0266(May 1998). https://doi.org/10.1002/(SICI)10970266(199805)19

Lau, A. K. W., \& Lo, W. (2015). Regional innovation system, absorptive capacity and innovation performance: An empirical study. Technological Forecasting and Social Change, 92, 99114. https://doi.org/10.1016/j.techfore.2014.11.005

Lerch, F., Seitz, G. M., \& Wagner, R. (2012). Absorptive capacity in collaborative technology transfer - a practice perspective on four cases in optics in the USA and Germany. 
International Journal of Knowledge Management Studies, 5(1/2), 66.

https://doi.org/10.1504/IJKMS.2012.051942

Lewin, A. Y., Massini, S., \& Peeters, C. (2011). Microfoundations of Internal and External Absorptive Capacity Routines. Organization Science, 22(1), 81-98. https://doi.org/10.1287/orsc. 1100.0525

Li, J. H., Huang, Q. B., \& Lin, L. (2014). Social capital and knowledge transfer in new service development: The front/back office perspective. Interdisciplinary Journal of Information, Knowledge, and Management, 9, 153-170. https://doi.org/10.28945 / 2073

Liao, J., Welsch, H., \& Stoica, M. (2003). Organizational Absorptive Capacity and Responsiveness: An Empirical Investigation of Growth-Oriented SMEs. Entrepreneurship Theory and Practice, 28(1), 63-86. https://doi.org/10.1111/1540-8520.00032

Lichtenthaler, U. (2009). Absorptive Capacity, Environmental Turbulence, and the Complementarity of Organizational Learning. Academy of Management Journal, 52(4), 822-846. https://doi.org/10.5465/amj.2009.43670902

Lin, C., Chang, S., \& Chang, C.-S. (2004). The impact of technology absorptive capacity on technology transfer performance. International Journal of Technology Transfer \& Commercialisation, 3(4), 1. https://doi.org/10.1504 / IJTTC.2004.005610

Martins, J. D. M. (2014). Operating Factors That Help to Improve Performance in Subsidiaries with Low Absorptive Capacity. Journal of East-West Business, 20(3), 162-183. https://doi.org/10.1080/10669868.2014.936575

Moja, L. P., Telaro, E., D’Amico, R., Moschetti, I., Coe, L., \& Liberati, A. (2005). Assessment of methodological quality of primary studies by systematic reviews: Results of the metaquality study cross sectional study. British Medical Journal, 330(7499), 1053-1055. https://doi.org/10.1136/bmj.38414.515938.8F

Mulamula, G., \& Amadi-Echendu, J. (2016). Leveraging Technology Transfer, Information Communication Technology and Capacity Building for Sustainable Development. 2015 IEEE International Conference on Engineering, Technology and Innovation/International Technology Management Conference, ICE/ITMC 2015, 1-11. 
https://doi.org/10.1109/ICE.2015.7438670

Newman, V., \& Chaharbaghi, K. (2000). Journal of Knowledge Management. The Study and Practice of Leadership, 4(1), 64-74. https://doi.org/10.1108/13673270010315966

Nieto, M., \& Quevedo, P. (2005). Absorptive capacity, technological opportunity, knowledge spillovers, and innovative effort. Technovation, 25(10), 1141-1157. https://doi.org/10.1016/j.technovation.2004.05.001

Nooteboom, B., \& Gilsing, V. (2006). Network embeddedness and the exploration of novel technologies: Technological distance, betweenness centrality and density. Politica de Investigación, 37(10), 1717-1731. https://doi.org/dx.doi.org/10.2139/ssrn.903743

Orlikowski, W. J. (2000). Using Technology and Constituting Structures : A Practice Lens for Studying Technology in Organizations, (May 2014). https://doi.org/doi.org/10.1287/orsc.11.4.404.14600

Pérez González, O. L., \& Portuondo Padrón, R. (1997). Evolución Histórica en las Concepciones sobre la Evaluación del Aprendizaje. Revista Pedagogía Universitaria. https://doi.org/10.15366/reice2017.15.1.007

Roberts, N., Galluch, P. S., Dinger, M., \& Grover, V. (2012). Absorptive capacity and information systems research : Review, synthesis, and directions. MIS Quarterly, 36(2), 625-648. https://doi.org/10.2307 / 41703470

Sambamurthy, V., Bharadwaj and, A., \& Grover, V. (2013). Shaping agility through digital options: Reconceptualizing the role of information technology in contemporary firm. Management Information Systems Research Center, University of Minnesota, 27(2), 237263. https://doi.org/10.2307 / 30036530

Sánchez Duarte, E. (2008). Las tecnologías de información y comunicación (TIC) desde una perspectiva social. Revista Electrónica Educare, XII, 155-162. https://doi.org/10.15359/ree.12-Ext.13

Serrat, O. (2017). Building a Learning Organization. Knowledge Solutions, (January), 1-38. https://doi.org/doi.org/10.1007/978-981-10-0983-9_11 
Stock, G. N., Greis, N. P., \& Fischer, W. A. (2001). Absorptive capacity and new product development. Journal of High Technology Management Research, 12(1), 77-91. https://doi.org/10.1016/S1047-8310(00)00040-7

Thorne, K. (2003). Blended Learning: How to Intergrate Online Learning and Traditional Learning. Kogan Page. Retrieved from http://books.google.com/books?id=xkBMgdG9up8C\&pgis=1\%0Ahttp://www.ncbi.nlm.nih. gov/pubmed/12971163

Van der Heiden, P., Pohl, C., Mansor, S., \& van Genderen, J. (2016). Necessitated absorptive capacity and metaroutines in international technology transfer: A new model. Journal of Engineering and Technology Management - JET-M, 41, 65-78. https://doi.org/10.1016/j.jengtecman.2016.07.001

Venkatesh, V., Davis, F. D., \& Venkatesh, V. (2000). A Theoretical Extension of the Technology Acceptance Model : Four Longitudinal Field Studies. Management Science Publication, (October 2018), 185-204. https://doi.org/10.1287/mnsc.46.2.186.11926

Wade, \& Hulland. (2004). Review: The Resource-Based View and Information Systems Research: Review, Extension, and Suggestions for Future Research. MIS Quarterly, 28(1), 107. https://doi.org/10.2307/25148626

Wang, C., \& Han, Y. (2011). Linking properties of knowledge with innovation performance: The moderate role of absorptive capacity. Journal of Knowledge Management. https://doi.org/10.1108/13673271111174339

Whittemore, R., Chao, A., Jang, M., Minges, K. E., \& Park, C. (2014). Methods for knowledge synthesis: An overview. Heart and Lung: Journal of Acute and Critical Care, 43(5), 453461. https://doi.org/10.1016/j.hrtlng.2014.05.014

Wiewiora, A., Chang, A., \& Smidt, M. (2020). Individual, project and organizational learning flows within a global project-based organization: exploring what, how and who. International Journal of Project Management, 38(4), 201-214. https://doi.org/10.1016/j.ijproman.2020.03.005

Zahra, S. A., \& George, G. (2002). Absorptive Capacity : a Review , and Extension. The 
Academy of Management Review, 27(2), 185-203.

https://doi.org/10.5465/APBPP.2000.5438568

Zollo, M., \& Winter, S. G. (2013). Deliberate Learning and the Evolution of Dynamic Capabilities. Organization Science, 13(3), 339-351.

https://doi.org/10.1287/orsc.13.3.339.2780 